\title{
Properties of $\mathrm{Si}_{-} \mathrm{SiO}_{2}$ Interface Traps \\ Due To Low-Energy $\mathrm{Ar}^{+}$Backsurface Bombardment \\ In n-Channel Nitrided MOSFETs
}

\author{
Charles Surya, W. Wang, W.K. Fong, and C.H. Chan \\ Department of Electronic Engineering \\ The Hong Kong Polytechnic University \\ Hong Kong \\ P.T. Lai \\ Department of Electrical and Electronic Engineering \\ University of Hong Kong \\ Hong Kong
}

\begin{abstract}
Flicker noise in backsurface gettered, nitrided n-channel MOSFETs is characterized over a wide range of temperatures and biases. The gettering time ranged from from 10 to 40 minutes. The noise power spectra for devices with different gettering times are compared to the ungettered devices which serve as the control. It is found that flicker noise is reduced by backsurface gettering for short gettering times. A rebound in the noise magnitude is observed for long gettering times. Investigations of the temperature dependencies of the noise power spectra indicates that the low-frequency noise arises from thermal activation of carriers to traps at the $\mathrm{Si}^{-\mathrm{SiO}_{2}}$ interface. Backsurface gettering results in the modification of the energy distribution of the interface traps, probably due to stress relaxation at the $\mathrm{Si}-\mathrm{SiO}_{2}$ interface.
\end{abstract}

\section{INTRODUCTION}

Nitridation of gate dielectrics in MOSFETs has been studied intensively for improvements in the oxide integrity and hot electron hardness [1]-[3]. This often leads to increase in $\mathrm{Si}_{-} \mathrm{SiO}_{2}$ interface states. Backsurface bombardment by ionic species is often used to getter metallic impurities and lattice defects, and could be a useful technique for gettering $\mathrm{Si}_{-} \mathrm{SiO}_{2}$ interface states. Recent work shows that low-energy gettering results in improvements in the carrier mobility which is speculated to arise from the reduction of $\mathrm{Si}_{-} \mathrm{SiO}_{2}$ interface traps.

Low-frequency excess noise in MOSFETs is highly sensitive to the presence of localized states[4]-[8]. In addition to being a important figure of merit of the device, it is also a useful technique for evaluating interface trap density, and is particularly useful in the energy range beyond the reach of conventional techniques such as $C-V$ and conductance measurements. In this paper we report experimental results on the properties of flicker noise in nitrided n-MOSFETs as a function of gettering time. We characterize the low-frequency excess noise over a wide range of gate and drain source voltages and from room temperature to about $90 \mathrm{~K}$. 


\section{EXPERIMENT}

Low-frequency noise is measured from nitrided $n$-channel MOSFETs fabricated on (100)-oriented wafers using polysilicon gate self-aligned technology. The gate oxide is about $240 \AA$ thick. The nitridation process is done by rapid thermal annealing of the transistors in $\mathrm{NH}_{3}$ at $1200{ }^{\circ} \mathrm{C}$ for 1 minute. Subsequently, the wafers are exposed to low-energy $(550 \mathrm{eV}) \mathrm{Ar}^{+}$beam for backsurface gettering. Gettering times of 10,20 , and 40 minutes have been used in this study and the devices are labeled as D10, D20 and D40 respectively. The control devices (D0) consist of ungettered n-MOSFETs with the same nitrided gate oxides.

Flicker noise is measured with the transistors current biased with passive sources. The devices are placed inside a liquid nitrogen continuous-flow cryostat for low temperature experiments. The averaged resistance noise power spectra normalized by the gate area are compared for devices with different gettering times. The result is shown in the table below:

\begin{tabular}{|c|c|}
\hline DEVICE & $\mathrm{S}_{\mathrm{RN}}(\mathrm{f}=1 \mathrm{kHz}) \Omega^{2} \mathrm{~cm}^{2} \mathrm{~Hz}^{-1}$ \\
\hline D0 & $9.23 \times 10^{-12}$ \\
\hline D10 & $8.64 \times 10^{-12}$ \\
\hline D20 & $8.15 \times 10^{-12}$ \\
\hline D 40 & $10.7 \times 10^{-12}$ \\
\hline
\end{tabular}

Table 1: Comparision of resistance noise power spectra with different gettering times.

Here we observe that the averaged resistance noise power spectral density decreases by up to $12 \%$ for a gettering time of 20 minutes. A rebound in the resistance noise is observed for gettering time of 40 minutes in which the average noise power spectra increased by $18 \%$ over the control devices.

The temperature dependences of the voltage noise power spectra are characterized. Our experimental data, for each gettering time, are shown in Fig. 1. It is observed that at low-temperature devices of different gettering times exhibit much bigger differences in the noise magnitudes. The temperature dependencies of the frequency exponent, $\gamma$, for devices with different gettering times are shown in Fig. 2.

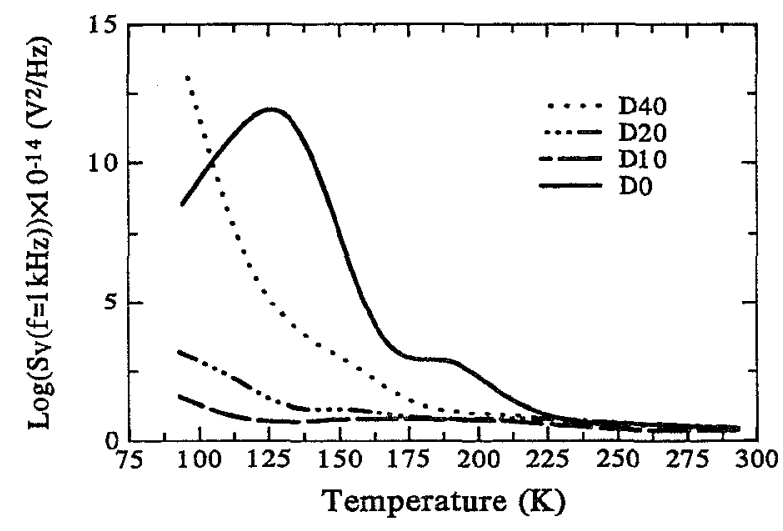

Fig. 1: Temperature dependence of $S_{V}(f=1 k H z)$.

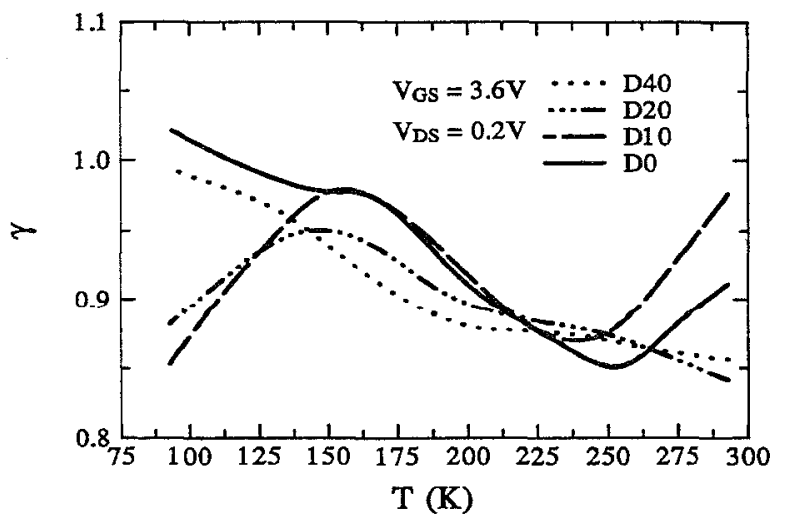

Fig. 2: Temperature dependence of $\gamma$. 


\section{DISCUSSIONS}

Our results show that $\mathrm{Ar}^{+}$backsurface gettering reduces the low-frequency excess noise for short gettering times. However, extended gettering times lead to the increase in the noise. Our experimental data compare very well with the results obtained from a recent study on the modification in the channel electron mobility as a function of gettering times on the same group of devices. A $10 \%$ improvement in the surface electron mobility for gettering times up to 20 minutes recorded by Lai et al[3] matches well with our observed improvements in the noise power spectra. Further gettering resulted in the degradation in both the carrier mobility and the low-frequency noise of the devices. The improvement in the carrier mobility was attributed to the reduction in both the interface states and the oxide fixed charge densities due to the relaxation in the interface stress by the gettering process. Since the low-frequency excess noise in MOSFETs arises from the capture and emission of carriers by interface states, a reduction in the interface states is accompanied by a corresponding reduction in the noise magnitude. In addition, both the electron mobility and the noise spectra improve by approximately the same amount clearly suggests that the two have the same physical origin. For long gettering times, degradation in both the electron mobility and the low-frequency excess noise is observed. It has been known that $1 / f$ noise in MOSFETs arises from the capture and emission of carriers at the $\mathrm{Si}_{-} \mathrm{SiO}_{2}$ interface. Therefore, the close resemblance in the dependencies of the noise magnitudes and the carrier mobilities on gettering clearly demonstrate that interface states play a key role in the variations of the two quantities.

Flicker noise has been shown to be a sensitive probe for $\mathrm{Si}_{-} \mathrm{SiO}_{2}$ interface traps at energy above the silicon conduction band edge at $E_{p}=-k_{B} T \ln \left(\tau_{0} \omega\right)$, where $k_{B}$ is the Boltzmann constant. These traps cannot be characterized using conventional techniques. Following the Dutta and Horn model[9] we obtained an estimate of the normalized trap distribution, $D_{i n}$, as given by the equation below:

$$
D_{i n}\left(E_{p}\right) \propto \frac{2 \omega}{\pi k_{B} T} S_{V}(\omega, T) .
$$

A plot of $D_{i n}(E)$ for different gettering times is shown in Fig. 3. Here we clearly observe a reduction in the trap density for short gettering times, however, a significant rebound in the trap density is seen for gettering time equals 40 minutes.

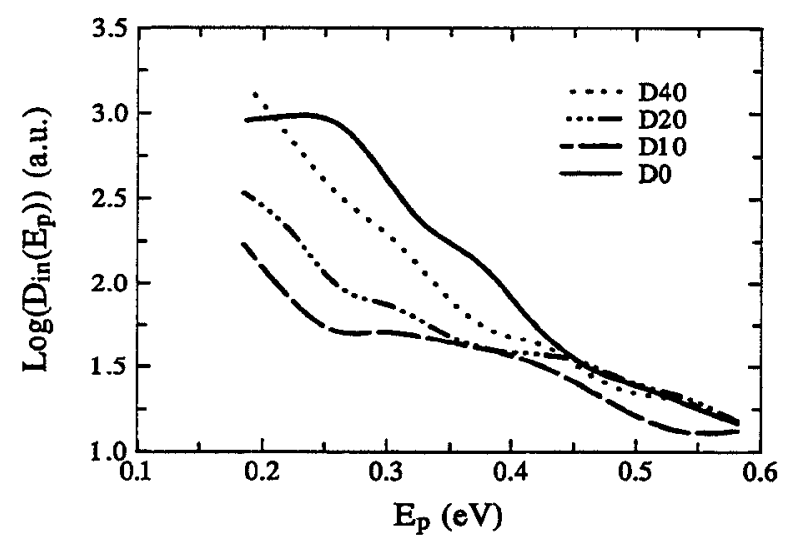

Fig. 3. Computed mormalizod $\mathrm{Si}_{\mathrm{S}} \mathrm{SiO}_{\mathrm{Z}}$ trap density as a function of energy 


\section{CONCLUSION}

In conclusion, we have conducted extensive studies on low-frequency excess noise in $\mathrm{Ar}^{+}$backsurface gettered, rapid thermal nitrided n-MOSFETs. Our results show that backsurface gettering is beneficial to the noise properties of the devices. Our experimental data indicated a $12 \%$ improvement in the resistance noise power spectral density for short gettering times. This improvement is attributed to stress relaxation at the $\mathrm{Si}_{-} \mathrm{SiO}_{2}$ interface. A rebound in the noise magnitude is observed upon further gettering of the devices. At 40 minutes gettering time, the averaged resistance noise power spectra is found to be $18 \%$ above that of the control devices. Based on the Dutta and Horn model we obtained the normalized trap distribution for different gettering times. A significant rebound in the trap density is observed for gettering time equals 40 minutes. This is probably due to over compensation of interface stress by gettering. To fully exploit the beneficial effects of backsurface gettering one needs to conduct more detailed experimentation on the relationships between the electrical properties of the devices and the gettering energy, gettering time as well as the annealing conditions.

\section{ACKNOWLEDGEMENTS}

This work is supported by the Unversity Research Grant, The Hong Kong Polytechnic University.

\section{References}

[1] S.K. Lai, J. Lee and V.K. Dham, IEDM Tech. Dig., 190 (1983).

[2] Z.H. Liu, P. Nee, P.K. Ko, C. Hu, C.G. Sodini, B.J. Gross, T.P. Ma, and Y.C. Cheng, IEEE Electron Device Lett. EDL-13, 41 (1992).

[3] P.T. Lai, Z. Xu, G.Q. Li, and W.T. Ng, IEEE Electron Device Lett. EDL-16, 354 (1995).

[4] C. Surya, and T.Y. Hsiang, Phys. Rev. B35, 6343 (1987).

[5] C. Surya, and T.Y. Hsiang, Solid-State Electron. 31, 959 (1988).

[6] Z.J. Ma, Z.H. Liu, Y.C. Cheng, P.K. Ko, and C. Hu, IEEE Trans. Electron Devices ED41, 2205 (1994).

[7] C. Surya, S.H. Ng, E.R. Brown, and P.A. Maki, IEEE Trans. Electron Devices ED41, 2016 (1994).

[8] S.H. Ng and C. Surya, Solid-State Electron. 35, 1213 (1992).

[9] P. Dutta and P.M. Horn, Rev. Mod. Phys. 53, 497 (1981). 